\title{
Acteoside Attenuates Oxidative Stress and Neuronal Apoptosis in Rats with Focal Cerebral Ischemia-Reperfusion Injury
}

\author{
Dongjian Xia, ${ }^{a, b}$ Zhuang Zhang, ${ }^{c}$ and Yuanli Zhao*,a \\ ${ }^{a}$ Department of Neurosurgery, Beijing Tian Tan Hospital, Capital Medical University; Beijing 100050, P. R. China: \\ ${ }^{b}$ Department of Neurosurgery, the First Affiliated Hospital, Jinzhou Medical University; Jinzhou 121001, P. R. \\ China: and ${ }^{c}$ Department of Neurology, the First Affiliated Hospital, Jinzhou Medical University; Jinzhou 121001, \\ P. R. China.
}

Received March 15, 2018; accepted August 19, 2018

\begin{abstract}
Acteoside (ACT) has been shown to exert antioxidant and neuroprotective effects in neurodegenerative diseases. However, the effect of ACT on cerebral ischemia-reperfusion (I/R) injury is not yet clear. In this study, we found that ACT administration reduced infarct volume and brain edema, and improved neurological deficits, as indicated by the decreased modified neurological severity score. Administration of ACT strikingly reduced oxidative stress, accompanied by decreased levels of reactive oxygen species and malondialdehyde and increased levels of superoxide dismutase and catalase in a rat model of middle cerebral artery occlusion/reperfusion (MCAO/R). Furthermore, ACT administration reduced the number of terminal deoxynucleotidyl transferase uridine 5'-triphosphate (UTP) nick-end labeling-positive cells in the cerebral cortex of ischemic side of MCAO/R rats, accompanied by downregulation of $\mathrm{B}$ cell lymphoma 2 (Bcl-2) associated X protein and cleaved caspase-3 proteins and upregulation of Bcl-2 protein. Additionally, ACT treatment inhibited the protein kinase $\mathrm{R} /$ eukaryotic initiation factor- $2 \alpha$ stress pathway in the brains of MCAO/R rats. Our results demonstrated that ACT attenuates oxidative stress and neuronal apoptosis in MCAO/R rats, suggesting that ACT may serve as a novel therapeutic candidate for the treatment of $I / R$ brain injury.
\end{abstract}

Key words acteoside; middle cerebral artery occlusion-reperfusion; oxidative stress; apoptosis

Stroke is one of the most common neurological disorders, and is the second largest cause of death and disability worldwide. ${ }^{1)}$ Based on its pathogenesis, stroke can be classified into 2 categories: ischemic stroke and hemorrhagic stroke. Ischemic stroke results from disturbances of the cerebral circulation, while hemorrhagic stroke occurs as a result of alterations to the blood vessels in the brain, which ultimately cause intraparenchymal or subarachnoid hemorrhage. ${ }^{2,3)}$ Of all adult strokes, approximately $80 \%$ are ischemic. ${ }^{4)}$ Currently, the best treatment for ischemic stroke is thrombolytic therapy. ${ }^{5)}$ However, restoration of blood circulation to the ischemic brain is not always effective. Conversely, it may cause an ischemia-reperfusion (I/R) injury. ${ }^{6}$ Thus, developing strategies to ease the injury caused by cerebral $I / R$ is the focal point of research at present.

Acteoside (ACT) or verbascoside is a caffeoyl phenylethanoid glycoside, which exists in multifarious plants, such as Cistanche spp., Pithecoctenium spp., and Plantago spp. $\mathrm{ACT}$ has been shown to exhibit neuroprotective activities in neurodegenerative diseases. For example, ACT protected SHSY5Y cells against amyloid-beta peptides $(\mathrm{A} \beta)_{25-35}$-induced cell injury through attenuating reactive oxygen species (ROS) production, reducing apoptosis, and improving mitochondrial membrane potential. $\left.{ }^{7}\right)$ Furthermore, ACT markedly repressed the aggregation of a 42-mer amyloid $\beta$ protein in a concentration-dependent manner. ${ }^{8)}$ In addition, ACT attenuated D-galactose-induced cerebral damage by inhibiting the increased expression of glial fibrillary acidic protein and neurotrophin-3.9) Besides, ACT mitigated amyloid $\beta$ peptide-induced cognitive dysfunction, neurotoxicity, and neurochemical disturbances via reducing amyloid protein deposition. ${ }^{10)}$ However, whether ACT exerts its neuroprotective effect in cerebral I/R injury remains elusive.

Protein kinase R (PKR), also known as double-stranded RNA (dsRNA)-activated protein kinase, has a C-terminal kinase domain and an N-terminal dsRNA-binding domain, which gives it pro-apoptotic functions. ${ }^{11)}$ PKR plays an important role in cellular responses to a variety of stimuli, such as oxidative stress, pathogens, and endoplasmic reticulum stress. ${ }^{12)}$ Eukaryotic initiation factor- $2 \alpha$ (eIF2 $\alpha$ ), the downstream signaling molecule of PKR, plays a key role in initiation of protein translation, thereby modulating cellular signaling. ${ }^{13)}$ Under normal conditions, PKR is expressed constitutively but is not functional. Under stress conditions, activated PKR directly phosphorylates eIF $2 \alpha$ at Ser51, which leads to inhibition of mRNA translation and ultimately results in apoptosis. ${ }^{14)}$ Recently, the PKR/eIF2 $\alpha$ pathway has been shown to be closely associated with the occurrence and development of various neurological and neuropsychiatric disorders. However, its role in cerebral I/R injury is still obscure.

In the present study, we found that ACT administration reduced infarct volume and brain water content and improved neurological deficits in middle cerebral artery occlusion/reperfusion $(\mathrm{MCAO} / \mathrm{R})$ rats. Moreover, our study concluded that ACT attenuates oxidative stress and neuronal apoptosis. These findings suggest that ACT may serve as a potential therapeutic agent for the treatment of cerebral I/R injury.

\section{MATERIALS AND METHODS}

Animals All animal experiments in this study were approved by the Institutional Animal Care and Use Committee of Beijing Tian Tan Hospital. Thirty male Wistar rats weighing 300-350g were purchased from Vital River Lab Animal 
Technology Co., Ltd. (Beijing, China), and kept under standard conditions of temperature $\left(22 \pm 2^{\circ} \mathrm{C}\right)$, relative humidity $(50 \pm 10 \%)$, and light $(12 / 12 \mathrm{~h}$ light/dark cycle). Rats had free access to a standard rodent diet ad libitum. Rats were distributed randomly as follows: Sham group: MCAO/R surgery was performed but no monofilament was advanced into the origin of the middle cerebral artery; $\mathrm{MCAO} / \mathrm{R}$ group: $\mathrm{MCAO} / \mathrm{R}$ surgery was performed; $\mathrm{MCAO} / \mathrm{R}+\mathrm{ACT}(10 \mathrm{mg} / \mathrm{kg} / \mathrm{d})$ group: $\mathrm{MCAO} / \mathrm{R}$ surgery was performed and ACT $(10 \mathrm{mg} / \mathrm{kg} / \mathrm{d}$; Catalog No. CFN97048; ChemFaces, Wuhan, China) was administered orally for 3 consecutive days; $\mathrm{MCAO} / \mathrm{R}+\mathrm{ACT}$ $(20 \mathrm{mg} / \mathrm{kg} / \mathrm{d})$ group: $\mathrm{MCAO} / \mathrm{R}$ surgery was performed and ACT $(20 \mathrm{mg} / \mathrm{kg} / \mathrm{d})$ was administered orally for 3 consecutive days; $\mathrm{MCAO} / \mathrm{R}+\mathrm{ACT}(40 \mathrm{mg} / \mathrm{kg} / \mathrm{d})$ group: $\mathrm{MCAO} / \mathrm{R}$ surgery was performed and ACT $(40 \mathrm{mg} / \mathrm{kg} / \mathrm{d})$ was administered orally for 3 consecutive days.

MCAO Surgery Rats were anesthetized by intraperitoneal injection of $10 \%$ chloral hydrate $(350 \mathrm{mg} / \mathrm{kg})$. Rectal and temporalis muscle temperatures were monitored and maintained at $37 \pm 0.5^{\circ} \mathrm{C}$. The rat was placed on its back and the right carotid artery was exposed through a neck incision. A 4-0 monofilament nylon thread with an expanded tip was then introduced into the internal carotid artery to block the origin of the middle cerebral artery. After $2 \mathrm{~h}$ of occlusion, the monofilament was gently withdrawn to commence reperfusion.

Brain Edema Measurement At $24 \mathrm{~h}$ after the last ACT treatment, brain edema was assessed using the dry/wet method. The brain of each rat was moved and placed in a preweighed dish to measure its wet weight (WW). Subsequently, the dish and the rat brains were desiccated in an oven at $110^{\circ} \mathrm{C}$ for $24 \mathrm{~h}$ to determine the dry weight (DW). Brain water content (\%) was determined using the following equation: $[(\mathrm{WW}-\mathrm{DW}) / \mathrm{WW}] \times 100$.

Measurement of Infarcted Volume To measure the infarct volume, rats were sacrificed and their brains were removed. Each brain was cut into $2 \mathrm{~mm}$ thick slices, and stained with 2,3,5-triphenyltetrazolium chloride (TTC; Beyotime, Shanghai, China) at $37^{\circ} \mathrm{C}$ for $20 \mathrm{~min}$. Subsequently, the slices were fixed in $10 \%$ formalin solution. The infarct volume was measured using ImageJ software (National Institutes of Health, NY, U.S.A.) and calculated as the sum of areas of 6 slices from each rat. Results were presented as a percentage of the infarct area.

Modified Neurological Severity Score (mNSS) At $24 \mathrm{~h}$ after the last ACT treatment, mNSS test was performed to assess neurological deficits. This test evaluated 4 aspects of neurological function, including motor, sensory, reflex, and balance functions. Neurological deficit was assessed using a 0-18 point rating scale (normal score: 0; maximal deficit score: 18). High scores indicated severe neurological deficit.

Enzyme-Linked Immunosorbent Assay (ELISA) The rat brains were homogenized in phosphate buffered saline (PBS) and centrifuged at $5000 \times \boldsymbol{g}$ for $5 \mathrm{~min}$ at $4^{\circ} \mathrm{C}$. The supernatant was used to determine the total ROS content using Rat
A

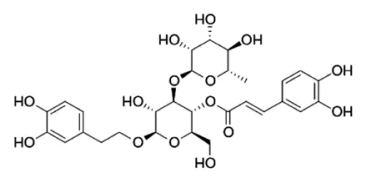

B
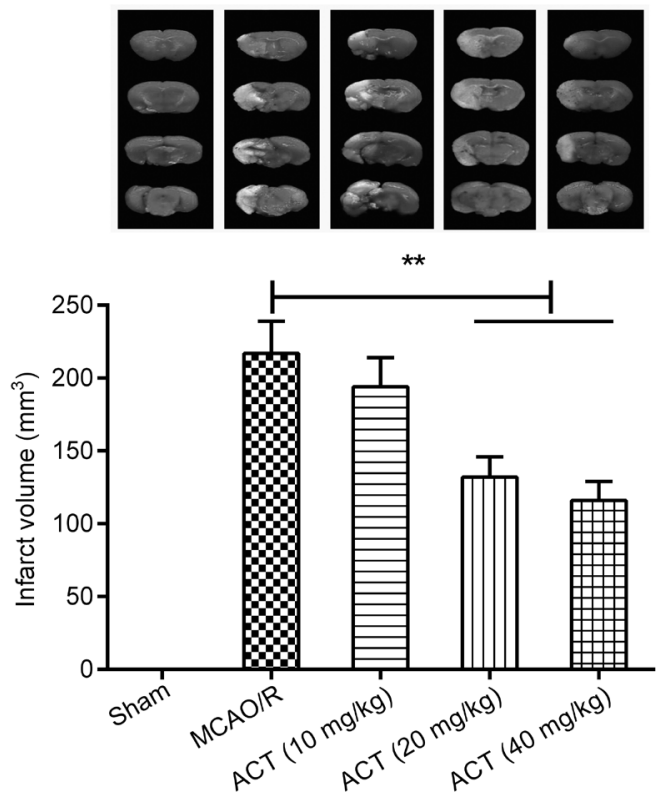

C

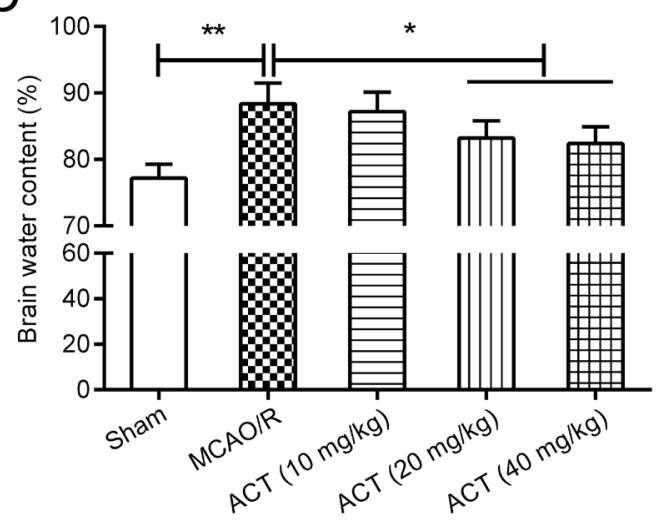

$\mathrm{D}$

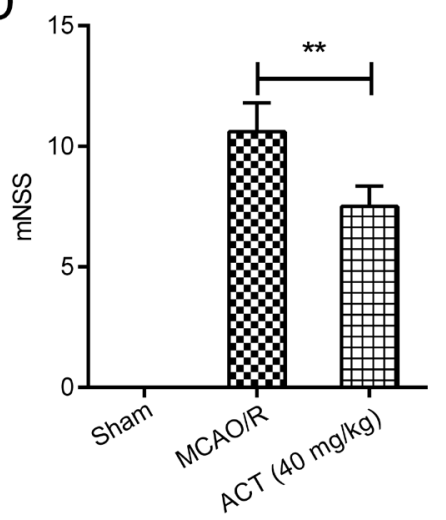

Fig. 1. Effect of ACT on Infarct Volume and Neurological Deficits in MCAO/R Rats

(A) The structure of ACT has been illustrated. After MCAO/R treatment, various doses of ACT (10, 20, and $40 \mathrm{mg} / \mathrm{kg} / \mathrm{d})$ were orally administered to MCAO/R rats for 3 consecutive days. Neurological deficits were assessed using TTC staining, standard wet-dry method, and mNSS test. (B and C) ACT treatment markedly reduced infarct volume and brain edema. (D) ACT administration improved neurological deficits, as indicated by reduction of $\mathrm{mNSS}$ scores. $* p<0.05, * * p<0.01$. 
ROS ELISA kit (Wuhan EIAab Science, Wuhan, China), in accordance with the manufacturer's instructions.

Measurement of Malondialdehyde (MDA), Superoxide Dismutase (SOD), and Catalase (CAT) Levels The levels of MDA, SOD, and CAT in the rat brains were measured using commercial assay kits (Solarbio, Beijing, China), in accordance with the manufacturer's instructions.

Terminal Deoxynucleotidyl Transferase Deoxyuridine Triphosphate (dUTP) Nick-End Labeling (TUNEL) Assay Apoptosis was detected using TUNEL assay. Briefly, the brain tissues sections were fixed in 4\% paraformaldehyde (Solarbio) for $15 \mathrm{~min}$, and then treated with $0.1 \%(\mathrm{v} / \mathrm{v})$ Triton X-100 (Solarbio) for $3 \mathrm{~min}$. Subsequently, the sections were incubated with TUNEL reaction mixture for $1 \mathrm{~h}$ at $37^{\circ} \mathrm{C}$ in the dark. Following this, sections were counterstained with 6-diamidino2-phenylindole (DAPI; Solarbio) for $5 \mathrm{~min}$ at $25^{\circ} \mathrm{C}$ in the dark. The number of TUNEL-positive cells were counted under a fluorescent microscope (Olympus, Tokyo, Japan). Results were expressed as percentage of TUNEL-positive cells.

Western Blotting The cerebral cortex of ischemic side was removed, grinded, and sonicated in lysis buffer at $4{ }^{\circ} \mathrm{C}$. Protein concentration of the lysate was determined using a BCA protein assay kit (Pierce, Rockford, IL, U.S.A.). Protein extracts $(50 \mu \mathrm{g})$ were resolved on $8 \%$ sodium dodecyl sulfatepolyacrylamide gels and then transferred to polyvinylidene difluoride membranes. After blocking with 5\% skim milk for
$2 \mathrm{~h}$ at room temperature, the membranes were probed with primary antibodies against $\mathrm{B}$ cell lymphoma 2 (Bcl-2) associated protein (Bax) (Abcam, Cambridge, MA, U.S.A.), Bcl-2 (Abcam), cleaved caspase-3 (Abcam), pPKR $\mathrm{Thr}_{446}$ (Abcam), PKR (Abcam), peIF $2 \alpha_{\text {Ser51 }}$ (Cell Signaling Technology, Beverly, MA, U.S.A.), eIF2 $\alpha$ (Cell Signaling Technology), and $\beta$-actin (Sigma-Aldrich, Louis, MO, U.S.A.) overnight at $4^{\circ} \mathrm{C}$. After rinsing in $\mathrm{PBS}$, the membranes were immunoblotted with horseradish peroxidase-conjugated secondary antibodies (Santa Cruz Biotechnology, Santa Cruz, CA, U.S.A.) for $1 \mathrm{~h}$ at room temperature. The membranes were developed using ECL reagents (Pierce), and protein bands were quantified using the Image J software.

Statistical Analysis All data analyses were performed using SPSS 20.0 software (SPSS, Chicago, IL, U.S.A.). Values were presented as mean \pm standard deviation (S.D.) representative of three independent experiments. Differences between groups were analyzed using one-way ANOVA followed by Tukey's post hoc test, and were considered statistically significant when $p<0.05$.

\section{RESULTS}

The Neuroprotective Effect of ACT against Cerebral I/R Injury in Rats The molecular structure of ACT is shown in Fig. 1A. To assess the neuroprotective effect of
A

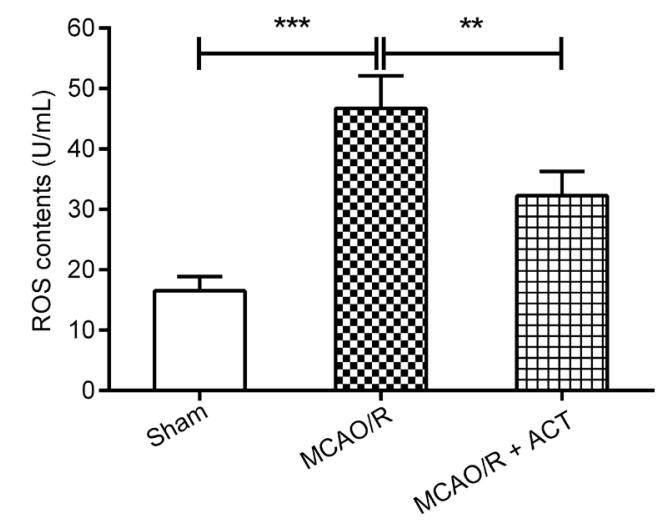

C

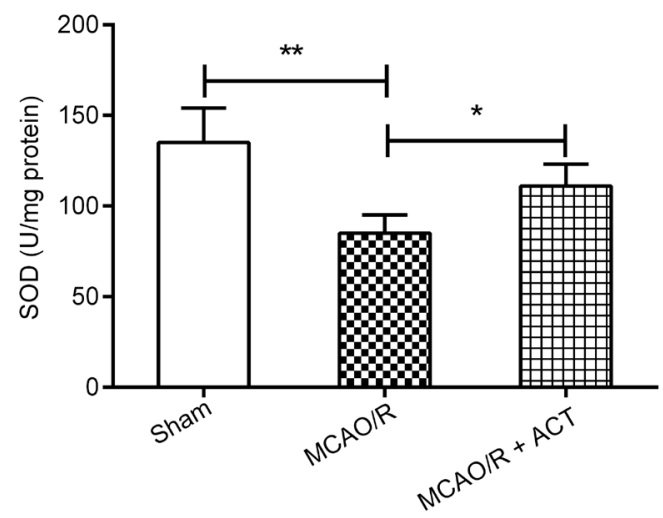

B

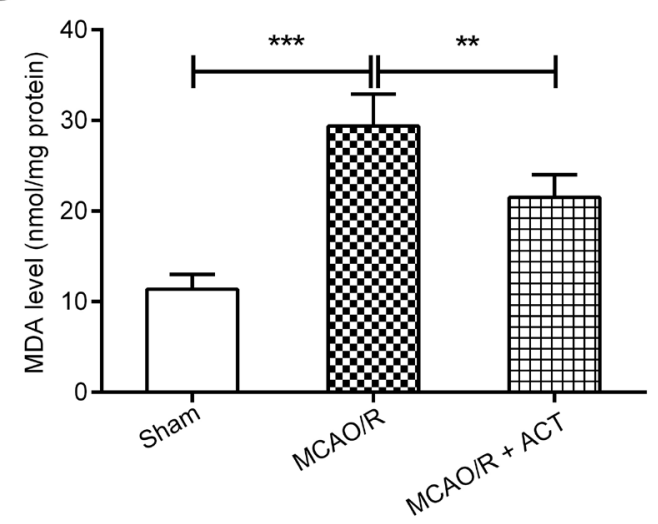

D

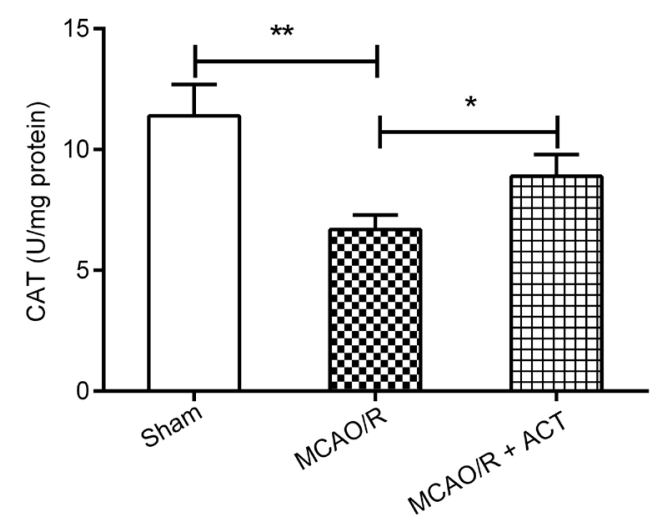

Fig. 2. ACT Attenuates Oxidative Stress in $\mathrm{MCAO} / \mathrm{R}$ Rats

After MCAO/R treatment, ACT ( $40 \mathrm{mg} / \mathrm{kg} / \mathrm{d}$ ) was orally administered to MCAO/R rats for 3 consecutive days. The levels of ROS (A), MDA (B), SOD (C), and CAT (D) in the cerebral cortex of ischemic side were then measured. ACT administration reduced the levels of ROS and MDA, and increased the levels of SOD and CAT in $\mathrm{MCAO} / \mathrm{R}$ rats. ${ }^{*} p<0.05, * * p<0.01, * * * p<0.001$. 
A

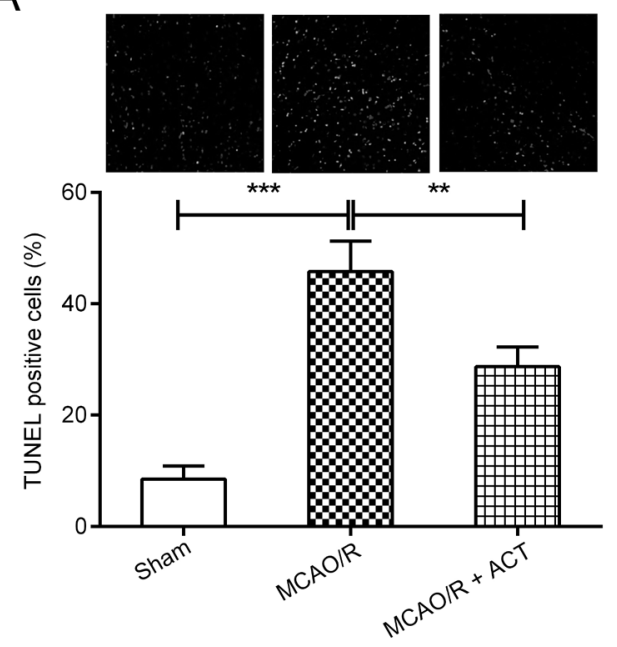

C
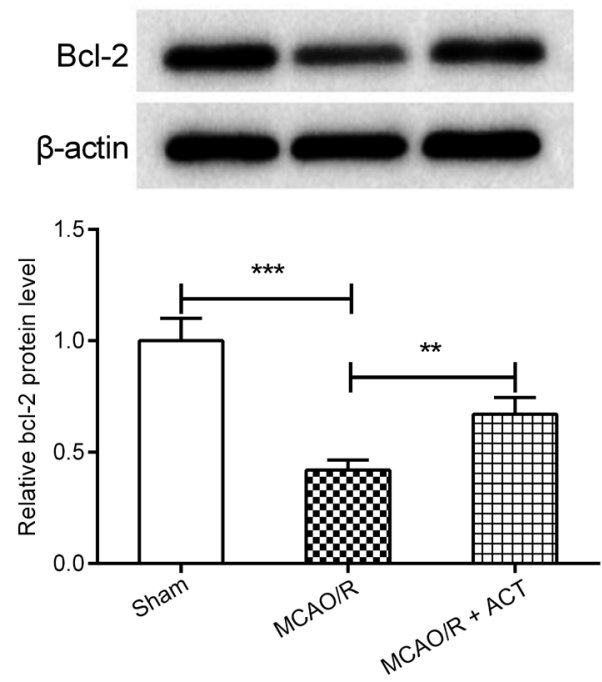

B

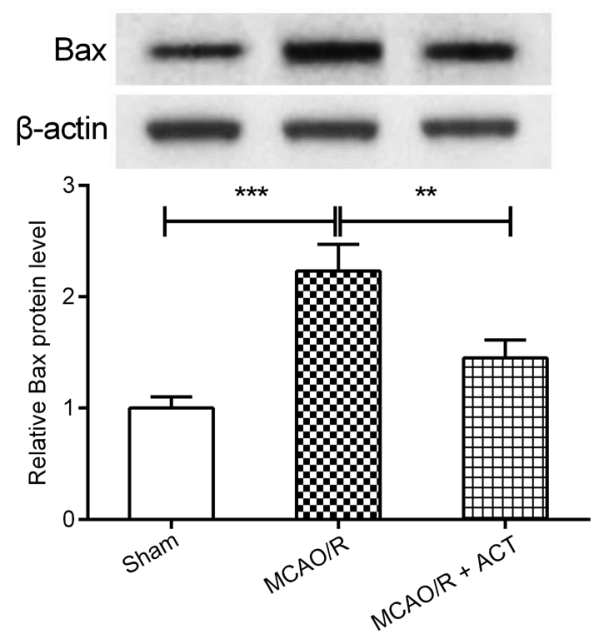

D
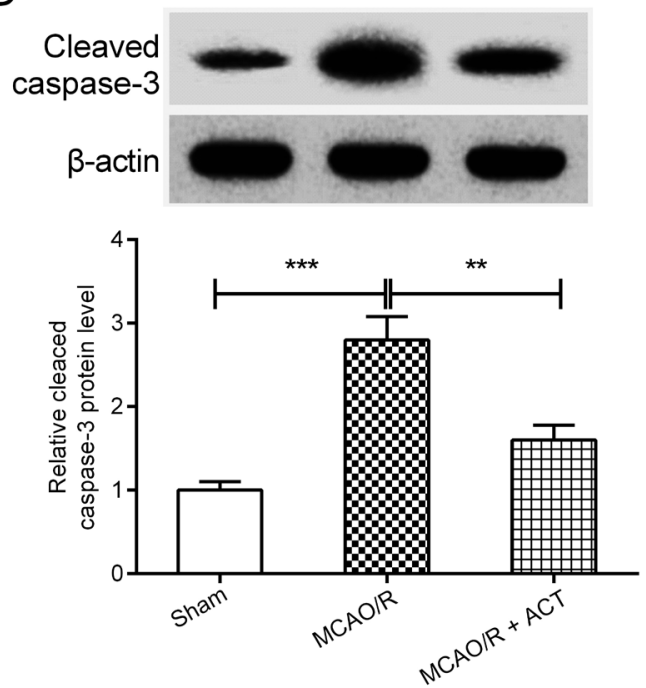

Fig. 3. ACT Prevents Neuronal Apoptosis in MCAO/R Rats

(A) Quantification of TUNEL-positive cells in the cerebral cortex of ischemic side of MCAO/R rats. ACT administration reduced the number of TUNEL-positive cells compared with the $\mathrm{MCAO} / \mathrm{R}$ group. Representative images of Western blot analysis and quantification of Bax (B), Bcl-2 (C), and cleaved caspase-3 (D) expression in the cerebral cortex of ischemic side of MCAO/R rats. ACT administration reduced the protein levels of Bax and cleaved caspase-3, but increased the Bcl-2 protein level in the cerebral cortex of ischemic side of $\mathrm{MCAO} / \mathrm{R}$ rats. ${ }^{* *} p<0.01,{ }^{* * *} p<0.001$.

ACT, various doses of ACT $(10,20$, and $40 \mathrm{mg} / \mathrm{kg} / \mathrm{d})$ were orally administered to $\mathrm{MCAO} / \mathrm{R}$ rats for 3 consecutive days. The infarcted area of the brain was assessed by TTC staining. Results showed that ACT administration (10, 20, and $40 \mathrm{mg} / \mathrm{kg} / \mathrm{d}$ ) resulted in reduced infarcted volume and brain edema compared to that in $\mathrm{MCAO} / \mathrm{R}$ rats, especially at a dose of $40 \mathrm{mg} / \mathrm{kg} / \mathrm{d}$ (Figs. 1B, 1C). Thus, a dose of $40 \mathrm{mg} / \mathrm{kg} / \mathrm{d}$ of ACT was selected for all following experiments. Correspondingly, ACT administration $(40 \mathrm{mg} / \mathrm{kg} / \mathrm{d})$ resulted in an obvious decrease in mNSS compared with that in MCAO/R rats (Fig. 1D). Therefore, our study revealed that ACT reduced infarcted volume and brain edema and improved neurological deficits in $\mathrm{MCAO} / \mathrm{R}$ rats.

ACT Attenuates Oxidative Stress in I/R Rats To investigate the effect of ACT on oxidative stress in MCAO/R rats, ROS, MDA, SOD, and CAT levels were measured. ROS and MDA levels were remarkably increased in the $\mathrm{MCAO} / \mathrm{R}$ group compared with those in the sham group, but decreased in the ACT group as compared with the MCAO/R group (Figs. 2A,
2B). Conversely, the levels of SOD and CAT were strikingly reduced in the $\mathrm{MCAO} / \mathrm{R}$ group compared with those in the sham group, but increased in the ACT group as compared with the MCAO/R group (Figs. 2C, 2D). Together, these data indicated that ACT attenuated oxidative stress in $\mathrm{MCAO} / \mathrm{R}$ rats.

ACT Prevents Neuronal Apoptosis in MCAO/R Rats To gain insights into the anti-apoptotic effect of ACT, we explored the effect of ACT on neuronal apoptosis in MCAO/R rats using TUNEL assay. As expected, an obvious increase in the number of TUNEL-positive cells was observed in the $\mathrm{MCAO} / \mathrm{R}$ group compared with that in the sham group, but this effect was blocked by ACT administration (Fig. 3A). To further elucidate the mechanism by which ACT elicits its anti-apoptotic effect, the expression levels of apoptosis-related proteins, including Bax, Bcl-2, and cleaved caspase-3 were determined by Western blotting. Results showed that the protein levels of Bax and cleaved caspase- 3 in the $\mathrm{MCAO} / \mathrm{R}$ group were strikingly higher compared to those in the sham group. 
A
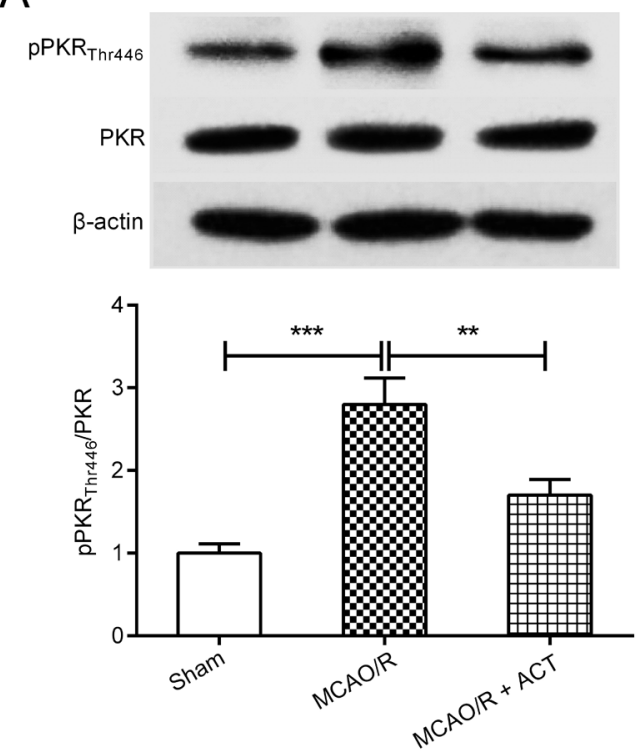

B

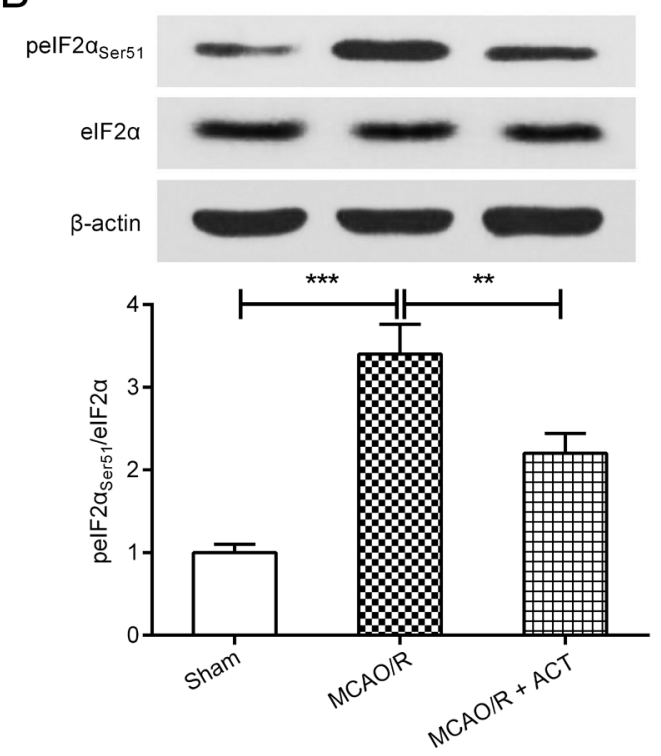

Fig. 4. ACT Inhibits the PKR/eIF2 $\alpha$ Stress Pathway

(A) Representative images of Western blot analysis and quantification of $\mathrm{pPKR}$ Thr446 and PKR expression in the cerebral cortex of ischemic side of MCAO/R rats. (B) Representative images of Western blot analysis and quantification of peIF $2 \alpha_{\text {Ser } 51}$ and eIF $2 \alpha$ expression in the cerebral cortex of ischemic side of MCAO/R rats. $* * p<0.01$, $* * * p<0.001$.

The protein levels of Bax and cleaved caspase-3 in the ACT group were lower compared to those in the $\mathrm{MCAO} / \mathrm{R}$ group (Figs. 3B, 3D). In contrast, the protein level of Bcl-2 was decreased in the MCAO/R group compared with that in the sham group, but increased in the ACT group compared with that in the MCAO/R group (Fig. $3 \mathrm{C}$ ). These results revealed that ACT protects neurons against $\mathrm{MCAO} / \mathrm{R}$-induced apoptosis.

ACT Inhibits the PKR/eIF2 $\alpha$ Stress Pathway To verify whether the neuroprotective effect of ACT was mediated by the PKR/eIF $2 \alpha$ stress pathway, the expression of $\mathrm{pPKR}_{\mathrm{Thr} 446}$, PKR, peIF $2 \alpha_{\text {Ser51 }}$, and eIF $2 \alpha$ in the brain of MCAO/R rats was examined by Western blotting. As shown in Figs. 4A and 4B, $\mathrm{MCAO} / \mathrm{R}$ treatment induced a striking increase in the protein levels of $\mathrm{pPKR} \mathrm{Thr}_{446}$ and peIF $2 \alpha_{\mathrm{Ser} 51}$. However, administration of ACT suppressed the MCAO/R-induced phosphorylation of PKR and eIF2 $\alpha$. Collectively, our findings suggested that ACT inhibited the PKR/eIF2 $\alpha$ stress pathway.

\section{DISCUSSION}

Oxidative stress has been implicated in brain damage and neuronal apoptosis during cerebral I/R. ${ }^{15)}$ Excessive production of ROS in neurons plays a crucial role in the development of cerebral I/R injury. Due to weak antioxidant enzyme activity, the brain is vulnerable to oxidative damage elicited by cerebral I/R injury, which ultimately leads to lipid peroxidation and ultimately neuronal apoptosis. ${ }^{16)}$ Oxidative stress causes disturbances in cerebral microcirculation and increases blood-brain barrier permeability, thereby resulting in brain edema. ${ }^{17)}$ Besides, in a previous study, oxidative stress led to disturbances in excitatory amino acid metabolism, resulting in excitotoxic injury. ${ }^{18)}$ Moreover, ROS can activate inflammatory cells through several mechanisms, which further aggravate brain injury. ${ }^{19)}$ Therefore, exploring drugs that attenuate oxidative stress-induced injury is very important. In this study, we found that ACT treatment reduced infarct volume and brain water content and decreased the mNSS in MCAO/R rats. Moreover, ACT administration mitigated oxidative stress, as indicated by decreased levels of ROS and MDA and increased levels of SOD and CAT.

Apoptosis is an autonomic ordered programmed cell death in order to maintain homeostasis, which is controlled by polygenes. $^{20,21)}$ Several lines of evidence have shown that apoptosis plays an important role in the pathogenesis of various human diseases, including cerebral I/R injury. During cerebral ischemia, both cyclooxygenase- 2 and inducible nitric oxide synthase are highly expressed, and induce activated microglial cells to generate ROS. ${ }^{22)}$ Overproduction of ROS leads to increased mitochondrial membrane permeability, resulting in translocation of Bax from the cytosol to the mitochondria and release of cytochrome $c$. $^{23)}$ Once released into the cytosol, cytochrome $c$ binds to apoptotic protease-activating factor 1 , which together with procaspase- 9 forms a complex termed the apoptosome. This complex activates procaspase-9 and -3 and ultimately results in neuronal apoptosis. ${ }^{24)}$ Therefore, targeting and preventing neuronal apoptosis in the brain appears to be a promising therapeutic strategy for the treatment of cerebral I/R injury. In the present study, ACT administration suppressed MCAO/R-induced neuronal apoptosis, as indicated by decreased Bax and cleaved caspase-3 levels and increased Bcl-2 levels, which was in accordance with a reduction in the number of TUNEL-positive cells.

There is growing evidence to suggest that the PKR/eIF $2 \alpha$ stress pathway is involved in the pathology of neurodegenerative disorders. For example, Mouton-Liger et al. demonstrated that the levels of pPKR and peIF2 $\alpha$ were increased in SH-SY5Y cells exposed to $\mathrm{H}_{2} \mathrm{O}_{2}{ }^{25)}$ Chang et al. showed that activation of the PKR/eIF $2 \alpha$ pathway was associated with neuronal apoptosis in Alzheimer's disease. ${ }^{26)}$ Zhang et al. suggested that gastrodin alleviated memory deficits in vivo and inhibited beta-amyloid converting enzyme 1 expression via inhibition of the PKR/eIF2 $\alpha$ pathway. ${ }^{27)}$ Ma et al. reported 
that chronic copper exposure might induce spatial memory impairment, selective loss of hippocampal synaptic proteins, and apoptosis through activation of the PKR/eIF2 $\alpha$ pathway. ${ }^{28)}$ Vaughn et al. demonstrated that inhibition of PKR delayed phosphorylation of eIF $2 \alpha$ and protected EV and K11 cells from tunicamycin-induced apoptosis in neuronal cells. ${ }^{29)}$ It has been reported that the apoptotic caspase-3, caspase-7, and caspase- 8 could cleave PKR, but the inflammatory caspase-1 and caspase-11 could not. During the apoptosis process, PKR activation and eIF2- $\alpha$ phosphorylation occur in a caspase-dependent manner. ${ }^{30)}$ Caspase-3 can cleave the alpha subunit of eIF-2, thus modulating the efficiency of protein synthesis. ${ }^{31)}$ In our study, a progressive enhancement of $\mathrm{pPKR}_{\mathrm{Thr} 446} / \mathrm{PKR}$ and peIF $2 \alpha_{\text {Ser } 51} /$ IF $2 \alpha$ ratio was observed in the cerebral cortex of ischemic side of $\mathrm{MCAO} / \mathrm{R}$ rats. Importantly, ACT administration inhibited the phosphorylation of PKR and eIF $2 \alpha$ at Thr446 and Ser51, respectively.

In conclusion, our study demonstrated that ACT administration reduced infarct volume and brain water content and improved neurological deficits. We also showed that ACT treatment attenuated the brain damage induced by cerebral I/R through mitigating oxidative stress and neuronal apoptosis. Our findings indicate that ACT may serve as a potential therapeutic agent for the treatment of cerebral I/R injury.

Conflict of Interest The authors declare no conflict of interest.

Supplementary Materials The online version of this article contains supplementary materials.

\section{REFERENCES}

1) Ribeiro PW, Cola PC, Gatto AR, da Silva RG, Luvizutto GJ, Braga GP, Schelp AO, de Arruda Henry MA, Bazan R. Relationship between dysphagia, national institutes of health stroke scale score, and predictors of pneumonia after ischemic stroke. J. Stroke Cerebrovasc. Dis., 24, 2088-2094 (2015).

2) Shigematsu K, Watanabe $Y$, Nakano H, Kyoto Stroke Registry Committee. Higher ratio of ischemic stroke to hemorrhagic stroke in summer. Acta Neurol. Scand., 132, 423-429 (2015).

3) Su X, Wang H, Kang D, Zhu J, Sun Q, Li T, Ding K. Necrostatin-1 ameliorates intracerebral hemorrhage-induced brain injury in mice through inhibiting RIP1/RIP3 pathway. Neurochem. Res., 40, 643$650(2015)$.

4) Gao S, Mo J, Chen L, Wang Y, Mao X, Shi Y, Zhang X, Yu R, Zhou X. Astrocyte GGTI-mediated Racl prenylation upregulates NF-kappaB expression and promotes neuronal apoptosis following hypoxia/ischemia. Neuropharmacology, 103, 44-56 (2016).

5) Sheth KN, Smith EE, Grau-Sepulveda MV, Kleindorfer D, Fonarow GC, Schwamm LH. Drip and ship thrombolytic therapy for acute ischemic stroke: use, temporal trends, and outcomes. Stroke, 46, 732-739 (2015)

6) Guell K, Bix GJ. Brain endothelial cell specific integrins and ischemic stroke. Expert Rev. Neurother., 14, 1287-1292 (2014).

7) Wang $\mathrm{H}, \mathrm{Xu} \mathrm{Y}$, Yan J, Zhao X, Sun X, Zhang Y, Guo J, Zhu C. Acteoside protects human neuroblastoma SH-SY5Y cells against beta-amyloid-induced cell injury. Brain Res., 1283, 139-147 (2009).

8) Kurisu M, Miyamae Y, Murakami K, Han J, Isoda H, Irie K, Shigemori $\mathrm{H}$. Inhibition of amyloid beta aggregation by acteoside, a phenylethanoid glycoside. Biosci. Biotechnol. Biochem., 77, 13291332 (2013)

9) Xiong L, Mao S, Lu B, Yang J, Zhou F, Hu Y, Jiang Y, Shen C,
Zhao Y. Osmanthus fragrans flower extract and acteoside protect against D-galactose-induced aging in an ICR mouse model. J. Med. Food, 19, 54-61 (2016).

10) Shiao YJ, Su MH, Lin $\mathrm{HC}, \mathrm{Wu}$ CR. Acteoside and isoacteoside protect amyloid $\beta$ peptide induced cytotoxicity, cognitive deficit and neurochemical disturbances in vitro and in vivo. Int. J. Mol. Sci., 18, 895 (2017).

11) Hugon J, Mouton-Liger F, Dumurgier J, Paquet C. PKR involvement in Alzheimer's disease. Alzheimers Res. Ther., 9, 83 (2017).

12) Tam CL, Hofbauer M, Towle CA. Requirement for protein kinase $R$ in interleukin-lalpha-stimulated effects in cartilage. Biochem. Pharmacol., 74, 1636-1641 (2007).

13) Xue B, Fitzgerald CA, Jin DZ, Mao LM, Wang JQ. Amphetamine elevates phosphorylation of eukaryotic initiation factor 2alpha (eIF2alpha) in the rat forebrain via activating dopamine D1 and D2 receptors. Brain Res., 1646, 459-466 (2016).

14) Nakamura T, Kunz RC, Zhang C, Kimura T, Yuan CL, Baccaro B, Namiki Y, Gygi SP, Hotamisligil GS. A critical role for PKR complexes with TRBP in immunometabolic regulation and eIF2alpha phosphorylation in obesity. Cell Reports, 11, 295-307 (2015).

15) Sies H. Oxidative stress: a concept in redox biology and medicine. Redox. Biol., 4, 180-183 (2015).

16) Xia Z, Chen Y, Fan Q, Xue M. Oxidative stress-mediated reperfusion injury: mechanism and therapies. Oxid. Med. Cell. Longev., 2014, 373081 (2014).

17) Bektas H, Wu TC, Kasam M, Harun N, Sitton CW, Grotta JC, Savitz SI. Increased blood-brain barrier permeability on perfusion CT might predict malignant middle cerebral artery infarction. Stroke, 41, 2539-2544 (2010).

18) Manzanero S, Santro T, Arumugam TV. Neuronal oxidative stress in acute ischemic stroke: sources and contribution to cell injury. Neurochem. Int., 62, 712-718 (2013).

19) Akhtar F, Rouse CA, Catano G, Montalvo M, Ullevig SL, Asmis R, Kharbanda K, Maffi SK. Acute maternal oxidant exposure causes susceptibility of the fetal brain to inflammation and oxidative stress. J. Neuroinflammation, 14, 195 (2017).

20) Tao T, Li CL, Yang WC, Zeng XZ, Song CY, Yue ZY, Dong H, Qian H. Protective effects of propofol against whole cerebral ischemia/reperfusion injury in rats through the inhibition of the apoptosis-inducing factor pathway. Brain Res., 1644, 9-14 (2016).

21) Li K, Ding D, Zhang M. Neuroprotection of osthole against cerebral ischemia/reperfusion injury through an anti-apoptotic pathway in rats. Biol. Pharm. Bull., 39, 336-342 (2016).

22) Yu S, Wang C, Cheng Q, Xu H, Zhang S, Li L, Zhang Q, Gu X, Ding F. An active component of Achyranthes bidentata polypeptides provides neuroprotection through inhibition of mitochondrialdependent apoptotic pathway in cultured neurons and in animal models of cerebral ischemia. PLOS ONE, 9, e109923 (2014).

23) Xing B, Chen H, Zhang M, Zhao D, Jiang R, Liu X, Zhang S. Ischemic postconditioning inhibits apoptosis after focal cerebral ischemia/reperfusion injury in the rat. Stroke, 39, 2362-2369 (2008).

24) Mendez DL, Akey IV, Akey CW, Kranz RG. Oxidized or reduced cytochrome $c$ and axial ligand variants all form the apoptosome in vitro. Biochemistry, 56, 2766-2769 (2017).

25) Mouton-Liger F, Paquet C, Dumurgier J, Bouras C, Pradier L, Gray F, Hugon J. Oxidative stress increases BACE1 protein levels through activation of the PKR-eIF2alpha pathway. Biochim. Biophys. Acta, 1822, 885-896 (2012).

26) Chang RC, Wong AK, Ng HK, Hugon J. Phosphorylation of eukaryotic initiation factor- $2 \alpha$ (eIF2 $\alpha$ ) is associated with neuronal degeneration in Alzheimer's disease. Neuroreport, 13, 2429-2432 (2002).

27) Zhang JS, Zhou SF, Wang Q, Guo JN, Liang HM, Deng JB, He WY. Gastrodin suppresses BACE1 expression under oxidative stress condition via inhibition of the PKR/eIF2alpha pathway in Alzheimer's disease. Neuroscience, 325, 1-9 (2016). 28) Ma Q, Ying M, Sui X, Zhang H, Huang H, Yang L, Huang X, 
Zhuang Z, Liu J, Yang X. Chronic copper exposure causes spatial memory impairment, selective loss of hippocampal synaptic proteins, and activation of PKR/eIF2alpha pathway in mice. J. Alzheimer's Dis., 43, 1413-1427 (2015).

29) Vaughn LS, Snee B, Patel RC. Inhibition of PKR protects against tunicamycin-induced apoptosis in neuroblastoma cells. Gene, 536, 90-96 (2014).
30) Saelens X, Kalai M, Vandenabeele P. Translation inhibition in apoptosis: caspase-dependent PKR activation and eIF2-alpha phosphorylation. J. Biol. Chem., 276, 41620-41628 (2001).

31) Satoh S, Hijikata M, Handa H, Shimotohno K. Caspase-mediated cleavage of eukaryotic translation initiation factor subunit 2alpha. Biochem. J., 342, 65-70 (1999). 